Textbook of Head and Neck Pathology 

Margaret S. Brandwein

\section{Textbook of Head and Neck Pathology}

Volume 1: Nose, Paranasal Sinuses, and Nasopharynx 


\section{Margaret S. Brandwein}

Erie County Medical Center

State University of New York at Buffalo

Buffalo

NY

USA

ISBN 978-3-319-33321-2

ISBN 978-3-319-33323-6 (eBook)

DOI 10.1007/978-3-319-33323-6

Library of Congress Control Number: 2016951646

(c) Springer International Publishing Switzerland 2016

This work is subject to copyright. All rights are reserved by the Publisher, whether the whole or part of the material is concerned, specifically the rights of translation, reprinting, reuse of illustrations, recitation, broadcasting, reproduction on microfilms or in any other physical way, and transmission or information storage and retrieval, electronic adaptation, computer software, or by similar or dissimilar methodology now known or hereafter developed.

The use of general descriptive names, registered names, trademarks, service marks, etc. in this publication does not imply, even in the absence of a specific statement, that such names are exempt from the relevant protective laws and regulations and therefore free for general use.

The publisher, the authors and the editors are safe to assume that the advice and information in this book are believed to be true and accurate at the date of publication. Neither the publisher nor the authors or the editors give a warranty, express or implied, with respect to the material contained herein or for any errors or omissions that may have been made.

Printed on acid-free paper

This Springer imprint is published by Springer Nature

The registered company is Springer International Publishing AG Switzerland 
To my children and grandchildren, the light of my eyes 



\section{Preface}

Each world contains a myriad of smaller worlds.

We choose the scale in which we dwell; perhaps, it selects us before we choose it. We pathologists are among a tiny cadre who can hear the whispering of glass slides. Hold a glass slide up to the air, you see a name, an institution, numbers, and tissue pieces. Put the glass on a micro- scope stage, and magic unfolds. The tissue holds worlds of stories, time lines of disease processes, symptoms, suffering, treatments, both successful and unsuccessful, and predictions. Imagine the worlds of information we glean from slides; now imagine the worlds we have yet to discover. This is our world. We only have to sit quietly, look, and listen to the whispering of slides. 



\section{Acknowledgment}

Special thanks to Dr. Nestor Rigual for

helpful review and feedback 



\section{Contents}

$1 \quad$ Sinonasal Tract - Anatomy and Histology ............................................................. 1

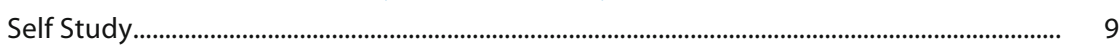

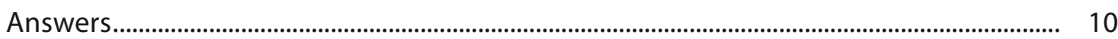

References....................................................................................................................... 10

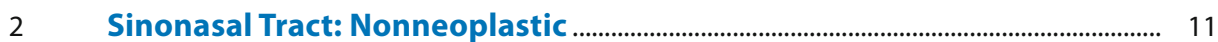

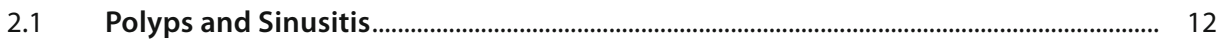

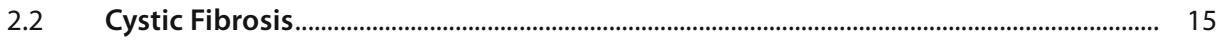

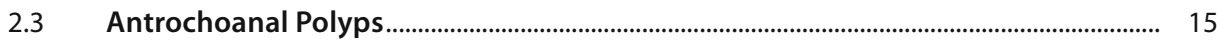

2.4 The Spectrum of Sinonasal Fungal Disease ................................................................... 16

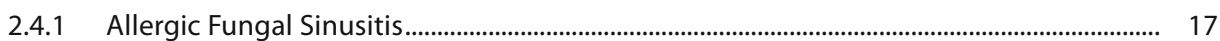

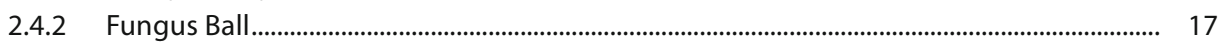

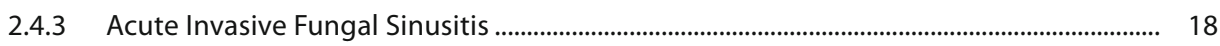

2.4.4 Granulomatous Invasive Fungal Disease..................................................................................... 20

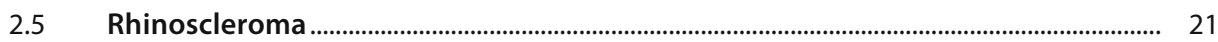

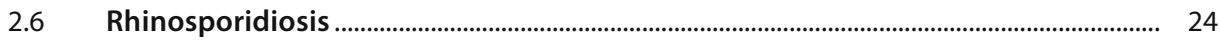

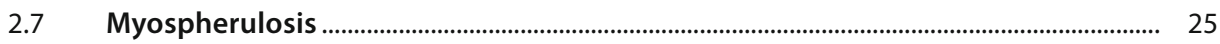

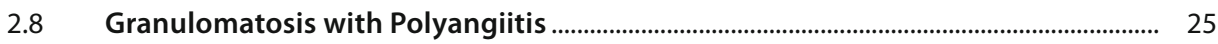

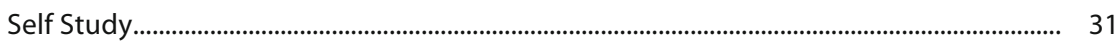

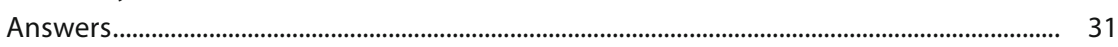

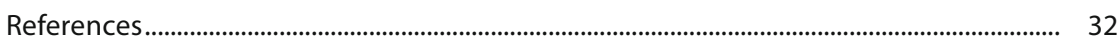

$3 \quad$ Sinonasal Tract - Benign .............................................................................................. 35

3.1 Respiratory Epithelial Adenomatoid Hamartoma (REAH) ............................................ 36

3.2 Sinonasal Seromucinous Hamartoma ................................................................................ 38

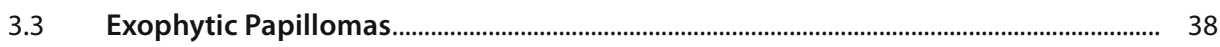

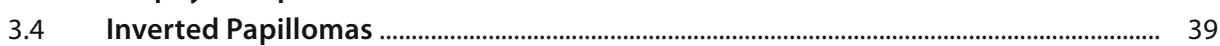

3.5 Oncocytic Schneiderian Papilloma ................................................................................ 43

3.6 Carcinoma-ex-inverted Papilloma .................................................................................... 44

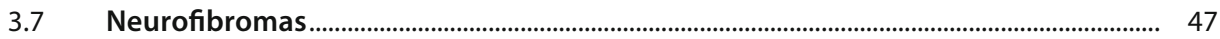

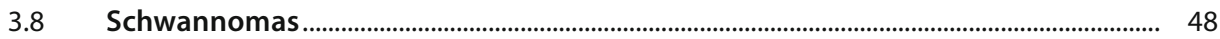

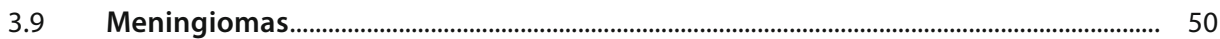

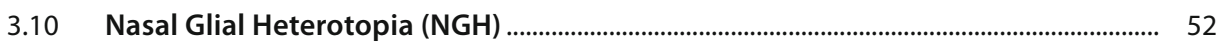

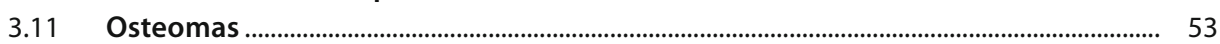

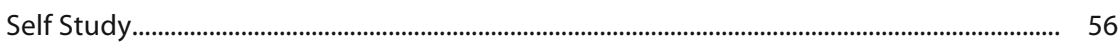

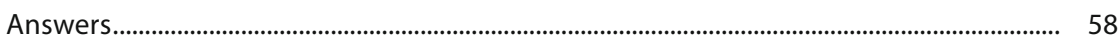

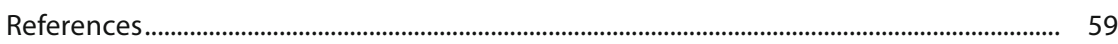

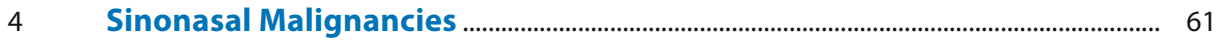

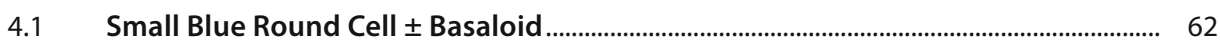

4.1.1 Sinonasal Undifferentiated Carcinoma (SNUC) ........................................................................ 62

4.1.2 BRD4-NUT Translocation Midline Carcinoma (Nut Midline Carcinoma, NMC) ................. 65

4.1.3 Sinonasal Human Papillomavirus-Related Adenoid-Cystic-Like Carcinoma...................... 66

4.2 Small Blue Round Cell \pm Epithelioid .................................................................................... 67

4.2.1 Transitional Variant Squamous Carcinoma ................................................................................ 67

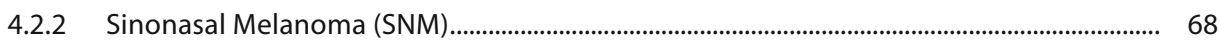

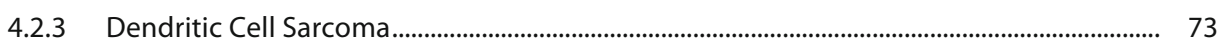


4.2.4 Extramedullary Plasmacytoma........................................................................................... 76

4.2.5 Sinonasal T-Cell and B-Cell Lymphomas.............................................................................

4.3 Small Blue Round Cell \pm Rhabdoid \pm Myxoid .............................................................. 80

4.3.1 Rhabdomyosarcoma.......................................................................................................... 80

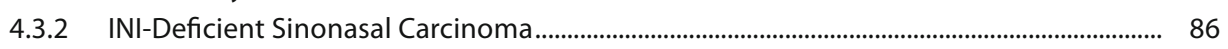

4.3.3 Sinonasal Low-Grade Myxoid Neoplasia .............................................................................. 86

4.4 Small Blue Round Cell Neuronal/Neuroendocrine ........................................................... 88

4.4.1 Olfactory Neuroblastoma.................................................................................................. 88

4.4.2 PNET (Primitive Nerve Sheath Tumor)/ES (Ewings' Sarcoma) or EFT (Ewing Family

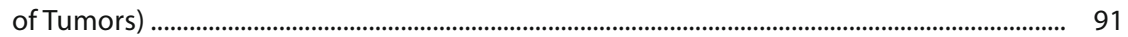

4.4.3 Sinonasal Neuroendocrine Carcinoma (SNEC) ...................................................................... 93

$4.5 \quad$ Glandular ........................................................................................................................... 96

4.5.1 Intestinal Type Adenocarcinoma (ITAC) .............................................................................. 96

4.5.2 Sinonasal Adenocarcinoma, (SNAC) Non Intestinal Type

(Not Otherwise Specified) .............................................................................................................. 100

4.5.3 Sinonasal Renal Cell-Like Adenocarcinoma .......................................................................... 101

4.5.4 Sinonasal Teratocarcinosarcoma (Malignant Teratoma, Teratocarcinoma) ........................ 103

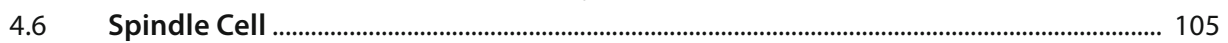

4.6.1 Sinonasal Hemangiopericytoma (Glomangiopericytoma)................................................ 105

4.6.2 Solitary Fibrous Tumor ........................................................................................................ 108

4.6.3 Fibrosarcoma/Myofibrosarcoma/Biphenotypic Sinonasal Sarcoma/Low-Grade

Sinonasal Sarcoma with Neural and Myogenic Features ...................................................... 110

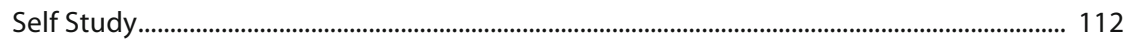

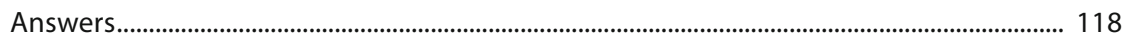

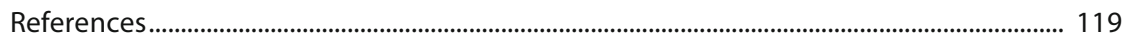

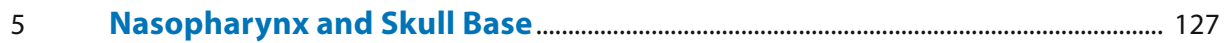

$5.1 \quad$ Anatomy and Histology ............................................................................................... 128

5.2 Tornwaldt's Cyst and Other Cysts .................................................................................. 132

$5.3 \quad$ Hairy Polyps and Teratomas ........................................................................................... 133

$5.4 \quad$ Nasopharyngeal Angiofibroma....................................................................................... 135

5.5 Salivary Gland Anlage Tumor (Nasopharyngeal Congenital

5.6 Craniopharyngioma (Rathke's Pouch Tumor) ................................................................ 141

5.7 Ectopic Pituitary Adenomas and Macroadenomas ……………………………………...... 142

$5.8 \quad$ Chordoma and Ecchordosis Physaliphora ....................................................................... 145

$5.9 \quad$ Nasopharyngeal Carcinoma .............................................................................................. 149

5.10 Post Transplant Lymphoproliferative Disease - Adenoids............................................. 154

5.11 Thyroid-Like Low-Grade Nasopharyngeal Papillary Adenocarcinoma....................... 155

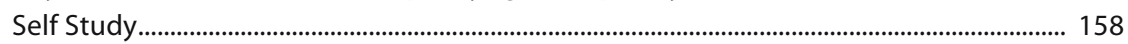

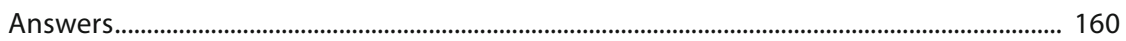

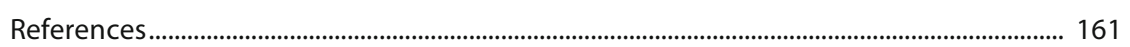

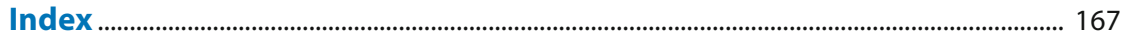

